\title{
Pneumothorax following Endobronchial Valve Therapy and Its Impact on Clinical Outcomes in Severe Emphysema
}

\author{
Daniela Gompelmann ${ }^{a} \quad$ Felix J.F. Herth ${ }^{a} \quad$ Dirk Jan Slebos ${ }^{b} \quad$ Arschang Valipour $^{c}$ \\ Armin Ernst ${ }^{d}$ Gerard J. Criner ${ }^{\mathrm{e}}$ Ralf Eberhardt ${ }^{a}$ \\ a Pneumology and Critical Care Medicine, Thoraxklinik, University of Heidelberg, and Translational Research Center \\ Heidelberg, Member of the German Center for Lung Research, Heidelberg, Germany; ${ }^{b}$ Department of Pulmonary \\ Diseases, University Medical Center Groningen, Groningen, The Netherlands; ' Ludwig Boltzmann Institute for COPD \\ and Respiratory Epidemiology, Otto Wagner Hospital, Vienna, Austria; ${ }^{d}$ Pulmonary, Critical Care and Sleep Medicine, \\ Caritas St. Elizabeth's Medical Center, Boston, Mass., and e Division of Pulmonary and Critical Care Medicine, Temple \\ University School of Medicine, Philadelphia, Pa., USA
}

For editorial comment see p. 452

\section{Key Words}

Emphysema $\cdot$ Endobronchial valves · Endoscopic lung volume reduction $\cdot$ Pneumothorax

\begin{abstract}
Background: Patients who achieve significant target lobe volume reduction (TLVR) following endobronchial valve (EBV) treatment may experience substantial improvements in clinical outcome measures. However, in cases of rapid TLVR, the risk of pneumothorax increases due to parenchymal rupture of the adjacent untreated lobe. Target lobe collapse may be more likely in EBV-treated patients who have low collateral ventilation. Objectives: The aim of this study was to evaluate the impact of pneumothorax on outcome following EBV treatment. Methods: Data from three prospective clinical trials (the US and European cohorts of VENT and the Multicenter Chartis study) were retrieved for the analysis. All patients had undergone chest $\mathrm{X}$-ray within $24 \mathrm{~h}$ of EBV implantation to explore the presence of pneumothorax. TLVR was assessed at either 30 (Chartis study) or 180 days (VENT), and clinical outcome measures (forced expiratory volume in $1 \mathrm{~s}\left(\mathrm{FEV}_{1}\right)$, St. George's Respiratory Question-
\end{abstract}

(c) 2014 S. Karger AG Basel

0025-7931/14/0876-0485\$39.50/0 naire (SGRQ) and 6-min-walk distance (6-MWD)) were assessed 180 days after implantation. Results: The overall rate of pneumothorax following valve therapy was $5.9 \%$ (25/421). Among these patients, $68 \%$ had a prolonged air leak for $>7$ days. However, patients who experienced a pneumothorax benefitted from EBV therapy, with a mean TLVR of $65 \%$ ( $n=$ 20). The mean percent change in $\mathrm{FEV}_{1}$ was $15 \pm 15 \%$, and the mean change in SGRQ was $-7 \pm 12$ points. Conclusions: Although pneumothorax is a complication of EBV placement, it does not appear to have a negative impact on clinical outcome in terms of $\mathrm{FEV}_{1}$ and health-related quality of life.

(c) 2014 S. Karger AG, Basel

\section{Introduction}

In recent years, endoscopic lung volume reduction has emerged as a new therapeutic option for patients with severe chronic obstructive pulmonary disease and emphysema. Among the different techniques of endoscopic lung volume reduction which are currently available, the implantation of valves is the most completely studied method to date. The first one-way valves were implanted by

\section{KARGER}

E-Mail karger@karger.com www.karger.com/res
Dr. med. D. Gompelmann

Pneumology and Critical Care Medicine, Thoraxklinik, University of Heidelberg Amalienstrasse 5

DE-69126 Heidelberg (Germany)

E-Mail daniela.gompelmann@med.uni-heidelberg.de 
Toma et al. [1] and Snell et al. [2] in 2003. These valves were placed in lobar or segmental bronchi leading to the most emphysematous lung lobe. While blocking inspired air and allowing trapped air to escape, the valves reduced hyperinflation thus optimizing diaphragm function and expiration. These changes in respiratory mechanics led to an improvement in pulmonary function and thus increased exercise capacity.

Various studies have confirmed the efficacy of valve placement in emphysema [3-5]. The first randomized controlled trial related to the implantation of endobronchial valves (EBV; Pulmonx, Inc., Palo Alto, Calif., USA) was VENT (Endobronchial Valves for Emphysema Palliation Trial) [5]. In this prospective trial, 321 patients with severe emphysema were randomly assigned to receive EBV treatment $(n=220)$ or optimum medical care $(n=101)$. Six months following valve implantation, statistically significant, but not clinically meaningful improvement in forced expiratory volume in $1 \mathrm{~s}\left(\mathrm{FEV}_{1}\right)$ and 6-min walk distance (6-MWD) were reported; similar results were obtained in the European cohort of the VENT (Euro-VENT) [6]. EBV treatment was performed on 111 patients, while 60 patients received standard medical care only. Significant improvements in $\mathrm{FEV}_{1}$, cycle ergometry and St. George's Respiratory Questionnaire (SGRQ) were reported.

Post hoc subgroup analyses of these prospective trials identified predictors of clinical response to valve placement. One of these predictive factors was the presence of a complete interlobar fissure, which was considered a surrogate for low interlobar collateral ventilation $(\mathrm{CV})$, as assessed by pre-interventional high-resolution computed tomography (HRCT). Therefore, assessment of CV prior to EBV placement is crucial to predict the patient's postoperative outcome. Besides HRCT fissure analysis, the Chartis ${ }^{\circledR}$ Pulmonary Assessment System (Pulmonx, Inc., Palo Alto, Calif., USA) provides a method for the assessment of CV during bronchoscopy $[7,8]$. This system enables the real time quantification of CV by isolating the targeted lobe using a dedicated catheter equipped with an inflatable balloon at its distal tip. In the multicenter Chartis trial, 96 patients were treated with EBV following CV measurement by the Chartis system [8]. The accuracy of the Chartis system to correctly predict the target lobe volume reduction (TLVR) was found to be $75 \%$.

Although the results of this therapeutic approach are persuasive, complications of EBV therapy need consideration. One adverse event is the development of pneumothorax following valve implantation (fig. 1) by parenchymal rupture that most commonly occurs in the adjacent untreated lung lobe. The risk of pneumothorax increases

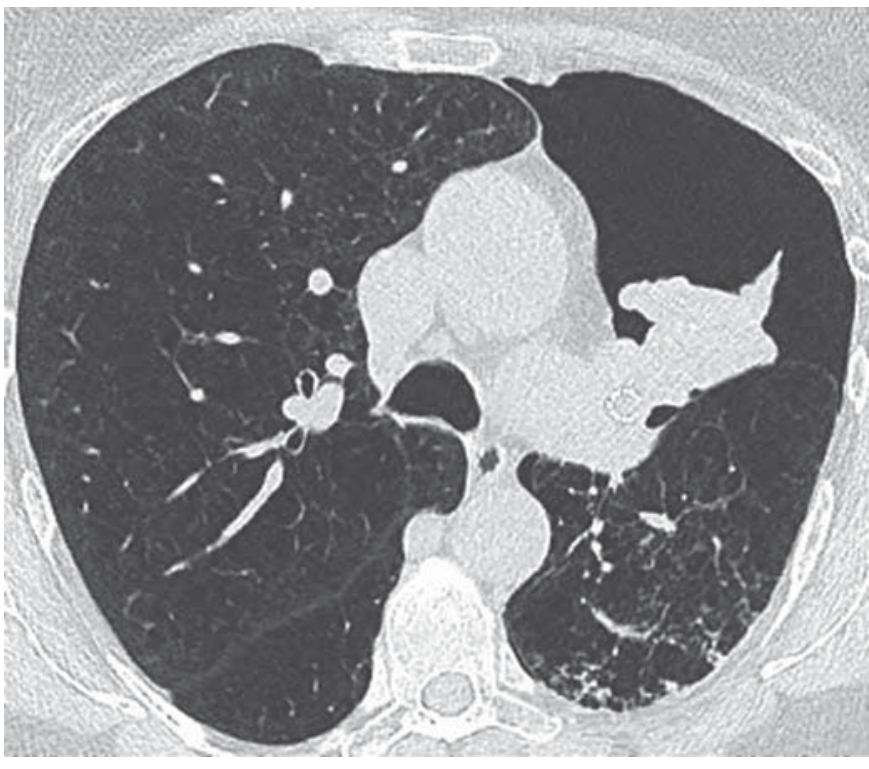

Fig. 1. Pneumothorax following successful EBV treatment in the left upper lobe with complete atelectasis of the treated lobe. In courtesy of C.P. Heussel, Thoraxklinik, University of Heidelberg, Germany.

particularly in cases of rapid TLVR. Thereby, patients with the greatest likelihood of TLVR after valve placement are at the highest risk of developing a pneumothorax. This retrospective analysis evaluated the impact of pneumothorax on outcome measures following EBV treatment.

\section{Materials and Methods}

In this retrospective analysis, clinical outcome of patients with pneumothorax following valve implantation was assessed in three prospective multicenter trials: VENT [a US study cohort (NCT00129584) and a European cohort (NCT00129584)] and the multicenter Chartis trial (NCT01101958).

The protocols of the different prospective trials were approved by their respective institutional review boards or ethics committees in the United States and Europe. In all studies, patients with severe emphysema were treated with EBV (Zephyr ${ }^{\circledR}$ EBV, Pulmonx, Inc., Palo Alto, Calif., USA) to completely occlude the most emphysematous lobe.

\section{Study Entry Criteria}

Trial designs and study entry criteria have been reported previously $[5,8]$. Briefly, patients with severe emphysema were enrolled in the study. In VENT, $\mathrm{FEV}_{1}$ between 15 and $45 \%$ and residual volume $(\mathrm{RV})>150 \%$ were further requirements for study inclusion. In the multicenter Chartis trial, $\mathrm{FEV}_{1}$ between 15 and $50 \%$ was an inclusion criterion.

VENT was a clinical controlled trial in which patients were randomly assigned to either an EBV group or a standard medical care group, whereas the Chartis trial was a single-arm study. In all trials, 
the patients assigned to the EBV arm received unilateral complete occlusion of the targeted lobe by EBV. In the multicenter Chartis trial, although CV was quantified by catheter-based measurement prior to EBV placement, this was not utilized as a screening criterion.

\section{Pneumothorax Evaluation}

All patients were hospitalized for a minimum of $24 \mathrm{~h}$ postoperatively. After EBV placement, a chest X-ray was performed to ascertain EBV location and to evaluate the presence of a pneumothorax. If dyspnea and/or thoracic pain occurred, another chest $\mathrm{X}$-ray was performed. In the case of pneumothorax, the subsequent procedure (chest tube insertion, video-assisted thoracoscopy or thoracotomy) was dependent on the extent of the pneumothorax and/or clinical symptoms. Furthermore, the median time to pneumothorax onset after EBV placement as well as the median time to pneumothorax resolution were recorded. All pneumothoraces occurring during the year following EBV placement were recorded.

Assessment of Fissure Integrity

To assess interlobar fissure integrity, HRCT scans performed at baseline were analyzed visually. A complete fissure was present when $>90 \%$ of the fissure was visible on at least one axis, which was determined by consensus of two independent readers at the HRCT core laboratory.

\section{Assessment of Clinical Outcome Changes}

At 180 days following the intervention, clinical follow-up comprised a pulmonary function test, a 6-min walk distance (6-MWD) test and a health-related quality of life score from the SGRQ. Furthermore, HRCT was performed to assess TLVR at the 30-day follow-up in the Chartis trial and the 180-day follow-up in VENT.

\section{Statistical Analysis}

Univariate analyses of the mean and median changes in TLVR, lung function parameters, 6-MWD and SGRQ of the patients who developed a pneumothorax during the 1st year following EBV placement were conducted.

\section{Results}

\section{Patient Inclusion}

As previously described, in the US VENT [5], 220 patients were randomly assigned to the EBV group and 214 of the 220 patients were treated. In the Euro-VENT, 111 patients received EBV placement [6]. In the multicenter Chartis study, 96 patients underwent CV measurement with the Chartis ${ }^{\circledR}$ Pulmonary Assessment System followed by EBV treatment. In summary, in these three prospective trials, 421 patients received unilateral complete occlusion of the targeted lobe by EBV.

\section{Pneumothorax Assessment}

In patients treated with EBV, the incidence of pneumothorax was 5.5\% (18/325) in VENT and 8.3\% (8/96) in the multicenter Chartis study 1 year after valve place-
Table 1. Characteristics of all patients with a pneumothorax

\begin{tabular}{lc}
\hline Patient characteristics $(\mathrm{n}=26)$ & Mean $\pm \mathrm{SD}$ \\
\hline Demographic characteristics & \\
$\quad$ Age, years & $63.0 \pm 7.1$ \\
Males, \% & $76.9 \%$ \\
BMI & $24.3 \pm 3.9$ \\
Pulmonary function & \\
FEV, liters & $0.98 \pm 0.3$ \\
FEV,$\%$ & $31.2 \pm 9.5$ \\
RV, liters & $5.42 \pm 1.8$ \\
RV, \% & $241.7 \pm 87.8$ \\
Exercise performance & \\
6-MWD, m & $340.3 \pm 106.0$ \\
Health-related quality of life & \\
SGRQ, points & $61.3 \pm 12.9$ \\
\hline
\end{tabular}

Table 2. Incidence of pneumothorax with respect to treatment lobe

\begin{tabular}{lll}
\hline Target lobe & $\begin{array}{c}\text { Total patient } \\
\text { population, } \mathrm{n}\end{array}$ & $\begin{array}{l}\text { Patients with } \\
\text { pneumothorax, n }\end{array}$ \\
\hline Right upper & 205 & $6(2.9 \%)$ \\
Left upper & 104 & $9(8.7 \%)$ \\
Left lower & 64 & $7(10.3 \%)$ \\
Right lower & 45 & $3(6.7 \%)$ \\
Right middle/right lower & 1 & 0 \\
Right middle & 2 & 0 \\
\hline
\end{tabular}

ment. Thus, the overall rate of pneumothorax was $6.2 \%$ (26/421). Table 1 shows the baseline characteristics of the patients who developed a pneumothorax. Of note, 1 patient in the US VENT experienced a left-sided pneumothorax 203 days following valve treatment in the right upper lobe. The reason for this contralateral pneumothorax was a lung biopsy performed for the assessment of lung nodules. This pneumothorax was not regarded as a consequence of EBV therapy and hence this case was excluded from the analysis. Thus, the rate of pneumothorax resulting from valve therapy was 5.9\% (25/421).

The incidence of pneumothorax was $10.3 \%$ in case of EBV placement in the left lower lobe followed by an incidence of $8.7 \%$ in case of EBV therapy in the left upper lobe (table 2). Therefore, the risk of pneumothorax seems to be higher following valve therapy in patients with a left-sided target lobe. The median time to pneumothorax onset following EBV placement was 2 days (0-272 days). The median duration of pneumothorax was 11 days (2-73 days).

Regarding the effect of fissure integrity, 17 of 25 patients $(68 \%)$ who developed a pneumothorax had a com- 
Table 3. Fissure integrity

\begin{tabular}{lll}
\hline Fissure integrity & $\begin{array}{l}\text { Total patient } \\
\text { population, } \mathrm{n}\end{array}$ & $\begin{array}{l}\text { Patients with } \\
\text { pneumothorax, } \mathrm{n}\end{array}$ \\
\hline Complete & 161 & $17(10.6 \%)$ \\
Incomplete & 234 & $7(3 \%)$ \\
Unknown & 26 & 1 \\
\hline
\end{tabular}

Table 4. TLVR (\%) in patients who experienced pneumothorax following valve therapy

\begin{tabular}{lll}
\hline Trial & Mean \pm SD & Median \\
\hline $\begin{array}{l}\text { VENT/Euro-VENT } \\
\text { (6-month follow-up; } \mathrm{n}=14)\end{array}$ & $60.2 \pm 38.1$ & 71.1 \\
\hline $\begin{array}{l}\text { Multicenter Chartis trial } \\
\text { (3-month follow-up; } \mathrm{n}=6)\end{array}$ & $74.5 \pm 30.0$ & 82.3 \\
\hline $\begin{array}{l}\text { VENT/Euro-VENT/ } \\
\text { Multicenter Chartis trial }(\mathrm{n}=20)\end{array}$ & $64.5 \pm 35.7$ & 78.8 \\
\hline
\end{tabular}

plete interlobar fissure and thus low interlobar CV. In 7 patients, an incomplete fissure was noted. In 1 patient, fissure integrity could not be assessed. Referring to the total patient population, the following data were obtained: $10.6 \%$ of EBV-treated patients with complete fissure experienced pneumothorax while only $3 \%$ of the patients with incomplete fissure developed a pneumothorax (table 3 ).

Seventeen of 25 patients $(68 \%)$ had a prolonged air leak for $>7$ days. In 21 of 25 patients (84\%), the pneumothorax resolved either under observation or following chest tube insertion. One patient (4\%) received a chest tube and was subsequently treated with two additional valves in the untreated ipsilateral lobe. These additional valves were removed after the air leak had resolved. In another patient (4\%), chest tube drainage was not successful to seal the fistula, and thus thoracotomy was required subsequently. In 1 patient (4\%), in whom pneumothorax did not resolve following chest tube drainage alone, valve removal, video-assisted thoracoscopy and additional thoracotomy were required to seal the air leak. One patient (4\%) was rehospitalized due to pneumothorax and concurrent pneumonia 12 days following valve placement. Chest drainage and antibiotic therapy were started. During the subsequent course, respiratory failure occurred, which required mechanical ventilation and valve explantation. After prolonged intensive care, the chest tubes were removed and the patient was weaned

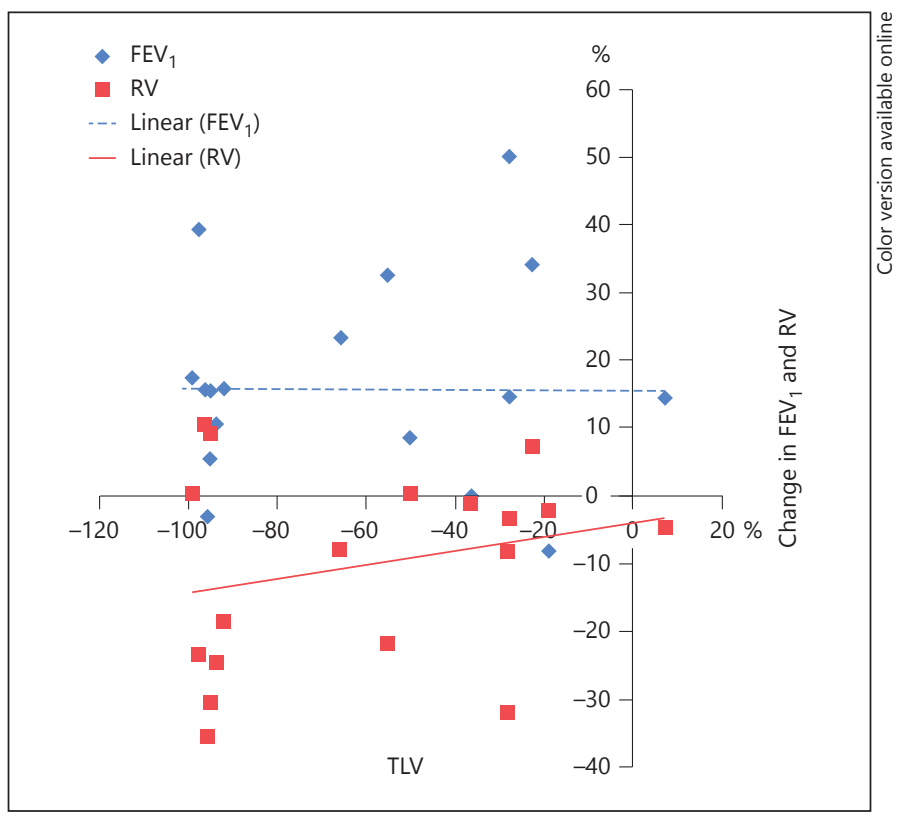

Fig. 2. Change of target lobe volume (TLV) and lung function parameters.

from the ventilator. Over time, however, the general condition of the patient worsened and the patient died 85 days following valve treatment.

Whereas the first-mentioned 2 patients with complicated pneumothorax experienced a good outcome with a TLVR of $>90 \%$, the latter 2 cases were not included in the efficacy analysis due to valve explantation.

\section{Pneumothorax and Clinical Outcome}

HRCT for TLVR assessment was available in 20 subjects. Patients with pneumothorax achieved high TLVR. In the VENT/Euro-VENT, a mean TLVR of $60 \pm 38 \%$ was observed at the 6-month follow-up; in the multicenter Chartis trial, a mean TLVR of $75 \pm 30 \%$ was found at the 1-month follow-up. In summary, EBV-treated patients experienced a mean TLVR of $65 \pm 36 \%$ in the three trials (table 4 ).

Regarding the 17 patients with a complete fissure, HRCT was not available for TLVR assessment in 3 patients. The remaining 14 patients had a mean TLVR of $58.4 \%$. 5 out of the 7 patients with incomplete fissures had a mean TLVR of $41.3 \%$.

In 20 of the 25 patients, a mean $\mathrm{FEV}_{1}$ change of $15 \pm$ $15 \%$ and a mean RV change of $-10 \pm 14 \%$ were observed at 180 days, respectively. Thereby, the improvement in lung function parameters, particularly the reduction in end expiratory lung volume, seemed to be associated with high TLVR (fig. 2). The 6-MWD increased slightly in the 

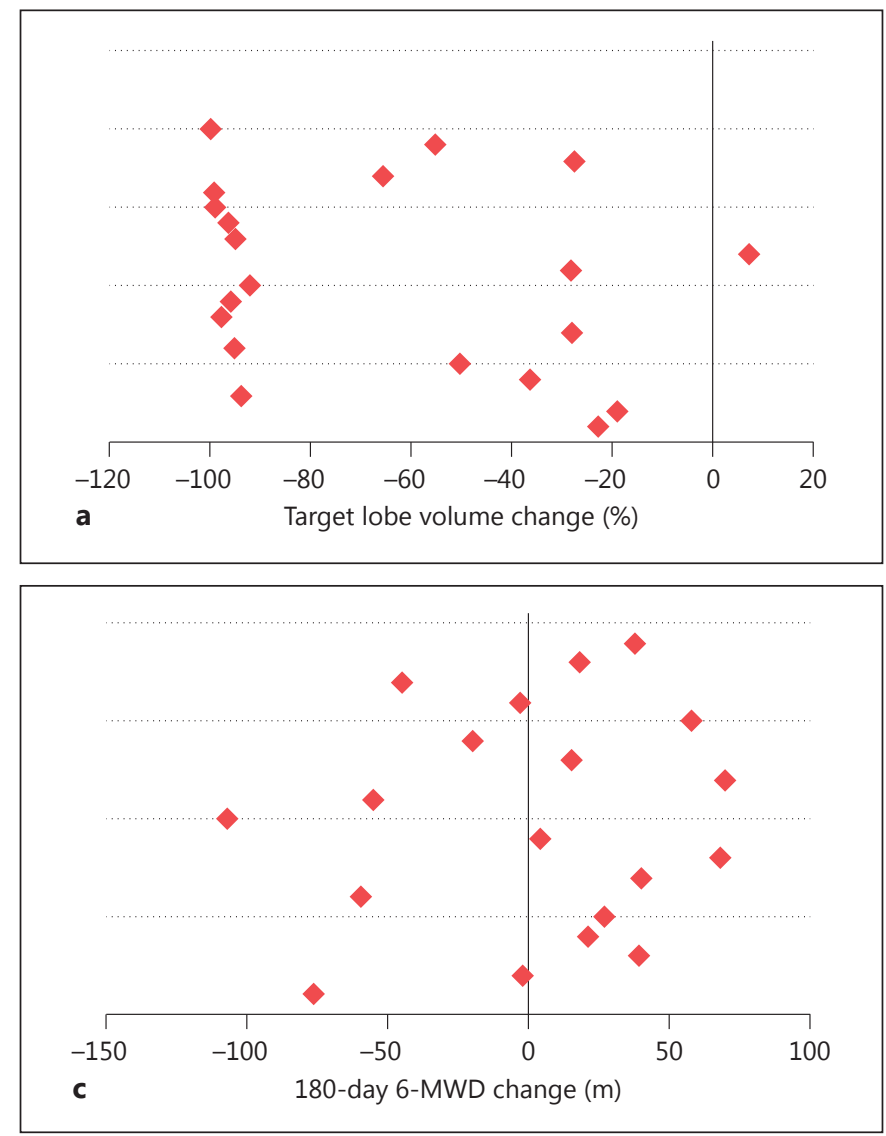

Fig. 3. Changes in TLV, $\mathrm{FEV}_{1}, 6-\mathrm{MWD}, \mathrm{RV}$ and SGRQ.

19 patients for whom it was available (mean 6-MWD change $2 \pm 50 \mathrm{~m}$ ). The SGRQ was assessed in 12 patients and demonstrated an improvement of $-7 \pm 12$ points. Tables 4 and 5 and figure 3 present the clinical outcomes.

Of patients with pneumothorax, $45 \%$ experienced a $>15 \%$ improvement in $\mathrm{FEV}_{1}$ at 6 months and $58 \%$ of patients had a $>4$-point improvement in SGRQ. Of the patients who developed pneumothorax, $18 \%$ experienced a $>15 \%$ improvement in 6-MWD.

Pneumothorax following EBV Therapy
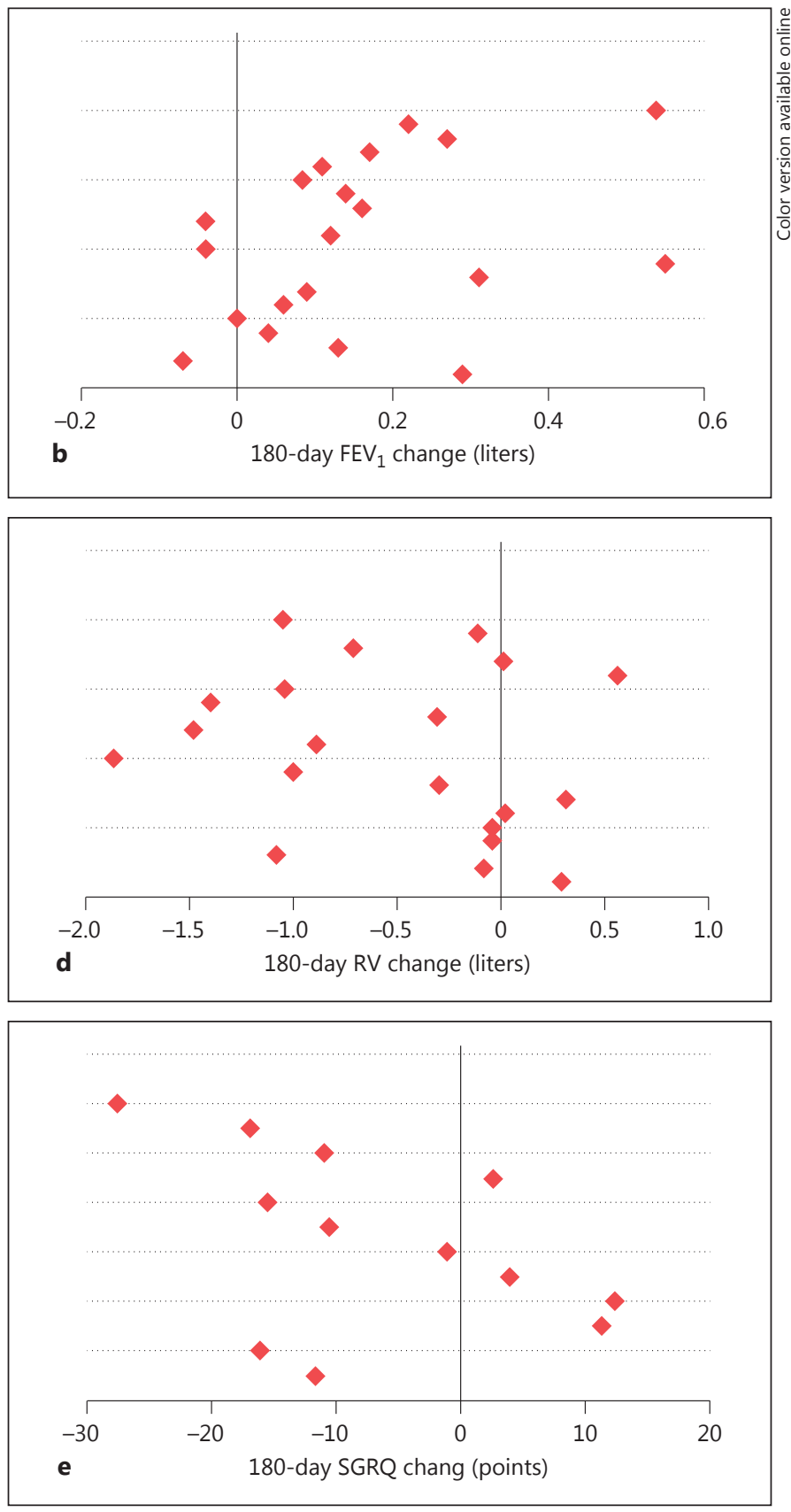

\section{Discussion}

In the last 10 years, endoscopic valve treatment has emerged as a new therapeutic option for severe emphysema. Several studies have confirmed a moderate improvement in lung function, exercise capacity and quality of life [3-7]. Post hoc analyses of these trials have revealed predictive factors that may be helpful in characterizing those patients who most likely benefit from this proce- 
Table 5. Clinical outcome of patients who experienced pneumothorax following valve therapy

\begin{tabular}{|c|c|c|c|}
\hline Parameters & Range & Mean \pm SD & Median \\
\hline \multicolumn{4}{|c|}{ Pulmonary function $(n=20)$} \\
\hline$\Delta \mathrm{FEV}_{1}$, liters & -0.07 to 0.55 & $0.16 \pm 0.2$ & 0.1 \\
\hline$\Delta \mathrm{FEV}_{1}, \%$ & -8.14 to 39.29 & $14.89 \pm 14.99$ & 14.62 \\
\hline$\Delta \mathrm{RV}$, liters & -1.86 to 0.56 & $-0.51 \pm 0.7$ & -0.3 \\
\hline$\Delta \mathrm{RV}, \%$ & -35.43 to 10.61 & $-10.31 \pm 14.23$ & -6.22 \\
\hline \multicolumn{4}{|c|}{ Exercise performance $(n=19)$} \\
\hline$\Delta 6-\mathrm{MWD}, \mathrm{m}$ & -107 to 70 & $1.61 \pm 50.2$ & 15.0 \\
\hline \multicolumn{4}{|c|}{ Health-related quality of life $(n=12)$} \\
\hline$\Delta$ SGRQ, points & -27.5 to 12.3 & $-6.69 \pm 12.3$ & -10.7 \\
\hline
\end{tabular}

180-day follow-up vs. baseline.

dure. These predictive characteristics include complete occlusion of the targeted lobe $[5,9]$ as well as lack of significant CV [7, 8].

However, there are also complications which are anticipated due to valve implantation, e.g. exacerbation of chronic obstructive pulmonary disease, pulmonary infection, pneumonia, hemoptysis, respiratory failure or cardiovascular events. Another major complication is the advent of pneumothorax following valve placement. It is likely that a parenchymal rupture of the ipsilateral, untreated lobe due to rapid expansion is the reason for the pneumothorax. Nevertheless, this presumption could not be confirmed as this is a retrospective analysis and the data set does not contain information regarding the specific anatomic location of the air leak.

However, one observation in support of this hypothesis is that $68 \%$ of the patients who developed pneumothorax following EBV treatment had a complete interlobar fissure that seems to be a surrogate of low CV. Furthermore, the incidence of pneumothorax in patients with a complete fissure was $10.3 \%$ compared to only $3 \%$ in patients with an incomplete fissure. As a targeted lobar collapse may be more likely to occur in subjects with low CV, fissure completeness may not only be the predictor for success but also for the development of a pneumothorax following EBV therapy. However, 32\% of the patients with pneumothorax and a mean TLVR of $41 \%$ had an incomplete fissure. This raises the so far not answered but frequently discussed question why some patients have success from EBV therapy even in the apparent presence of an incomplete fissure as a surrogate of interlobar $\mathrm{CV}$. The multicenter Chartis trial also demonstrated that $17.2 \%$ of the patients with proof of $\mathrm{CV}$ benefit from valve therapy.
One possible explanation for the development of pneumothorax in patients with incomplete fissure or high CV is that these patients had only a small fissural gap or collateral channels with a small caliber imitating a high $\mathrm{CV}$, which, however, is not significant in the physiological state.

Despite pneumothorax being a complication that requires further interventions, the patients achieved substantial lobar volume reduction and in most cases excellent clinical outcomes. The mean TLVR was $65 \pm 36 \%$, thus reaching a threshold associated with improvements in both lung function and health-related quality of life [10]. However, a pneumothorax per se does not lead to a good outcome, but it seems to be simply an indicator of an insignificant $\mathrm{CV}$, as discussed above. Comparing the patients who developed pneumothorax to the subgroup of all patients with complete fissure in VENT, similar results can be observed: $45 \%$ of the patients with pneumothorax experienced a $>15 \%$ improvement in $\mathrm{FEV}_{1}$ compared to $42.6 \%$ of all patients with a complete fissure in VENT. Of the pneumothorax patients, $18 \%$ had a $6-\mathrm{MWD}$ change of $15 \%$ compared to $20.6 \%$ of the patients with complete fissure in VENT. Therefore, pneumothorax does not appear to have a negative impact on clinical outcome in terms of $\mathrm{FEV}_{1}$ and health-related quality of life.

Despite the clinical benefit observed in the patients who developed a pneumothorax, it should be stressed that pneumothorax presents a major complication particularly in patients with severe emphysema. A prolonged air leak for $>7$ days was present in $68 \%$ of the patients. In $16 \%$ of the patients who developed pneumothorax, further interventional procedures, e.g. video-assisted thoracoscopy, pleurodesis or thoracotomy, were necessary. Furthermore, it must be borne in mind that although the mean change in TLVR, lung function parameters, exercise capacity and health-related quality of life in patients with pneumothorax is encouraging, there are some patients who experience no improvement in clinical outcome measures.

One of the 25 patients (4\%) who developed a pneumothorax died 85 days following EBV placement. It must be assumed that this complication, which followed the valve treatment, led to longer immobility and the need for intensive care and hospitalization, which worsened the patient's overall general condition. However, this death was probably not related to pneumothorax or valve placement itself, as the patient recovered completely from pneumothorax following valve removal and chest drainage.

During the three trials, no pneumothorax guidelines existed and thus different approaches were used to treat 
a pneumothorax. The results of these trials demonstrate that close monitoring following valve implantation is crucial.

In our trial, the median time to the onset of a pneumothorax following EBV placement was 2 days. Fifteen patients experienced pneumothorax within $48 \mathrm{~h}$, and 2 further patients within $74 \mathrm{~h}$. Therefore, patients should be hospitalized for a minimum of $72 \mathrm{~h}$ after the intervention. Chest $\mathrm{X}$-ray should be performed $2 \mathrm{~h}$ after valve insertion and repeated if symptoms occur. In case a pneumothorax develops, the following regimen is proposed: a watchand-wait strategy is feasible in the event of small pneumothoraces among asymptomatic patients, whereas chest tube insertion is required in symptomatic patients who experience a significant pneumothorax. In the case of prolonged fistulas, the explantation of one valve should be considered, thus facilitating re-expansion of the lung. Consequently, the decision to opt for valve removal depends on fistula volume, soft tissue emphysema and clin- ical status. If the air leak still persists, all valves should be removed. A video-assisted thoracoscopy with pleurodesis has to be discussed in case of a persistent air leak although all valves are explanted.

In this analysis, 8 patients developed late-onset pneumothorax. All were rehospitalized and required a chest tube. Therefore, it is crucial to inform and educate the patients on the risk of pneumothorax.

In summary, endoscopic valve implantation presents an effective treatment in patients with severe emphysema and low CV. As a pneumothorax is an anticipated complication of EBV therapy, especially in those without CV in the lobe targeted for treatment, close monitoring following the intervention is necessary. Nevertheless, patients who develop a pneumothorax experience clinically significant improvements. Therefore, the event of pneumothorax seems to be an indicator of a successful EBV therapy. The benefits of valve therapy must be weighed carefully against the increased risk of pneumothorax.

\section{References}

$>1$ Toma TP, Hopkinson NS, Hillier J, Hansell DM, Morgan C, Goldstraw PG, Polkey MI, Geddes DM: Bronchoscopic volume reduction with valve implants in patients with severe emphysema. Lancet 2003;361:931-933.

$>2$ Snell GI, Holsworth L, Borrill ZL, Thomson KR, Kalff V, Smith JA, Williams TJ: The potential for bronchoscopic lung volume reduction using bronchial prostheses: a pilot study. Chest 2003;124:1073-1080.

-3 Venuta F, de Giacomo T, Rendina EA, Ciccone AM, Diso D, Perrone A, Parola D, Anile M, Coloni GF: Bronchoscopic lungvolume reduction with one-way valves in patients with heterogenous emphysema. Ann Thorac Surg 2005;79:411-416; discussion 416-417.
-4 Wan IY, Toma TP, Geddes DM, Snell G, Williams T, Venuta F, Yim AP: Bronchoscopic lung volume reduction for end-stage emphysema: report on the first 98 patients. Chest 2006;129:518-526.

5 Sciurba FC, Ernst A, Herth FJF, Strange C, Criner GJ, Marquette CH, Kovitz KL, Chiacchierini RP, Goldin J, McLennan G; VENT Study Research Group: A randomized study of endobronchial valves for advanced emphysema. N Engl J Med 2010;363:1233-1244.

$\checkmark 6$ Herth F, Noppen M, Valipour A, Leroy S, Vergnon J-M, Ficker JH, Egan E, Gasparini S, Agusti C, Homes-Higgin D, Ernst A; International VENT Study Group: Efficacy predictors of endoscopic lung volume reduction with Zephyr valves in a European cohort. Eur Respir J 2012;39:1334-1342.

7 Gompelmann D, Eberhardt R, Michaud G, Ernst A, Herth FJ: Predicting atelectasis by assessment of collateral ventilation prior to endobronchial lung volume reduction: a feasibility study. Respiration 2010;80:419-425.
8 Herth FJ, Eberhardt R, Gompelmann D, Ficker JH, Wagner M, Ek L, Schmidt B, Slebos DJ: Radiological and clinical outcomes of using Chartis $^{\mathrm{TM}}$ to plan endobronchial valve treatment. Eur Respir J 2013;41:302-308.

$>9$ Eberhardt R, Gompelmann D, Schuhmann M, Reinhardt H, Ernst A, Heussel CP, Herth FJ: Complete unilateral versus partial bilateral endoscopic lung volume reduction in patients with bilateral lung emphysema. Chest 2012; 142:900-908.

10 Valipour A, Herth FJF, Burghuber OC, Criner G, Vergnon J-M, Goldin J, Sciurba F, Ernst A; VENT Study Group: Target lobe volume reduction and COPD outcome measures after endobronchial valve therapy. Eur Respir J 2014;43:387-396. 